\title{
Hyperbolic Metrics on Surfaces with Boundary
}

\author{
Melanie Rupflin ${ }^{1}$ \\ Received: 21 August 2019 / Published online: 7 March 2020 \\ (c) The Author(s) 2020
}

\begin{abstract}
We discuss an alternative approach to the uniformisation problem on surfaces with boundary by representing conformal structures on surfaces $M$ of general type by hyperbolic metrics with boundary curves of constant positive geodesic curvature. In contrast to existing approaches to this problem, the boundary curves of our surfaces $(M, g)$ cannot collapse as the conformal structure degenerates which is important in applications in which $(M, g)$ serves as domain of a PDE with boundary conditions.
\end{abstract}

Keywords Uniformisation · Hyperbolic surfaces · Surfaces with boundary · Geometric flows

Mathematics Subject Classification 30F10 $\cdot 53 \mathrm{C} 44$

\section{Introduction}

Given a surface $M$, there are many interesting questions with regard to representing a given conformal structure by a Riemannian metric.

A classical question in this context, for $M=S^{2}$ known as Nirenberg's problem, asks what functions can occur as Gauss curvatures of such metrics on closed surfaces, and over the past decades this problem has been studied by many different authors, we refer in particular to $[1,6,13,24,25]$ as well as the more recent work of [2,7] and the references therein for an overview of existing results. We also note that the corresponding problem on surfaces with boundary was investigated in [8] .

Another classical problem in this context, but of a quite different flavour, is to ask how to 'best' represent a given conformal structure by a Riemannian metric. For closed surfaces this problem is addressed by the classical uniformisation theorem that allows us to represent every conformal structure by a (unique for genus at least 2) metric of constant Gauss curvature $K_{g} \equiv 1,0,-1$, while for complete surfaces this

\footnotetext{
Melanie Rupflin

rupflin@maths.ox.ac.uk

1 Mathematical Institute, University of Oxford, Oxford OX2 6GG, UK
} 
problem was addressed by Mazzeo and Taylor in [15]. On surfaces with boundary, Osgood, Philips and Sarnak introduced in [17] two different notions of uniformisation, with uniform metrics of type I characterised by having constant Gauss curvature and geodesic boundary curves, while uniform metrics of type II are flat and have boundary curves of constant geodesic curvature. The corresponding heat flows were analysed by Brendle in [3], who proved that these flows admit global solutions which converge to the corresponding uniform metric in the given conformal class. As observed by Brendle in [4], for the two different types of uniform metrics introduced in [17], only one of the terms on the left-hand side of the Gauss-Bonnet formula

$$
\int_{M} K d v_{g}+\int_{\partial M} k_{g} d S_{g}=2 \pi \chi(M)
$$

gives a contribution and so the two types of uniform metrics can be seen the opposite ends of a whole family of metrics for which all terms in the above formula have the same sign. Brendle [4] proved also in this more general setting that solutions of the corresponding heat flows exist for all times and converge, now to metrics with $K_{g} \equiv \bar{K}$ and $k_{g} \equiv \bar{k}$, where the signs of $\bar{K}$ and $\bar{k}$ both agree with the sign of $\chi(M)$. We note that the same restriction on the signs of the curvatures is also present in the work of Cherrier [8].

Here we propose an alternative way of representing conformal structures on surfaces of general type with boundary which is motivated by applications to geometric flows, such as Teichmüller harmonic map flow [19,23] or Ricci-harmonic map flow [16], in which the surface $(M, g)$ plays the role of a time dependent domain on which a further PDE is solved. For this purpose the described ways of uniformisation on surfaces with boundary suffer the serious drawback that a degeneration of the conformal structure, which can occur even for curves of metrics with finite length, can lead to a degeneration of the metric near the boundary curves, with boundary curves turning into punctures in the limit, so that the very set on which the boundary condition is imposed can be lost.

To resolve this problem, we propose to represent conformal classes on surfaces of general type instead by hyperbolic metrics for which each boundary curve is a curve of positive constant geodesic curvature, chosen so that each of the boundary curves gives a fixed positive contribution to the Gauss-Bonnet formula. As we shall see below, this alternative approach has the advantage that the resulting metrics will remain well controlled near the boundary even if the conformal class degenerates in a way that would cause the boundary curves of the corresponding uniform metrics of type I or II to collapse. The existence of a unique representative of each conformal class with these desired properties is ensured by our first main result.

Theorem 1.1 Let $M$ be a compact oriented surface of genus $\gamma$ with boundary curves $\Gamma_{1}, \ldots, \Gamma_{k}$ and negative Euler characteristic and let $d>0$ be any fixed number. Then for any conformal structure $\mathrm{c}$ on $M$, there exists a unique hyperbolic metric $g$ compatible with c for which

$$
\left.k_{g}\right|_{\Gamma_{i}} \cdot L_{g}\left(\Gamma_{i}\right) \equiv d \text { on } \Gamma_{i} \text { for every } i=1 \ldots k
$$


Denoting by $\mathcal{N}_{-1}^{d}$ the set of all hyperbolic metrics on $M$ satisfying (1.1), we furthermore have that for every $g \in \mathcal{M}_{-1}^{d}$ and every $i \in\{1, \ldots, k\}$, there exists a unique simple closed geodesic $\gamma_{i}$ in the interior of $M$ which is homotopic to $\Gamma_{i}$ and this geodesic is surrounded by a collar neighbourhood $\mathrm{C}\left(\gamma_{i}\right)$ that is described in Lemma 3.2 and its length is related to the length of the corresponding boundary curve by

$$
L_{g}\left(\Gamma_{i}\right)^{2}-L_{g}\left(\gamma_{i}\right)^{2}=d^{2} .
$$

For hyperbolic surfaces with boundary curves of constant geodesic curvature $k_{g}$, the relation (1.2) between the lengths of a boundary curve and the corresponding geodesic is equivalent to (1.1) and we note that (1.1) implies that the area of the region enclosed by $\Gamma_{i}$ and $\gamma_{i}$ is always equal to $d$. We remark that the quantity (1.2) appears also naturally if one studies horizontal curves of metrics on closed surfaces, that is curves of hyperbolic metrics which move $L^{2}$ orthogonally to the action of diffeomorphisms, as for such curves, the analogue of (1.2) is valid at the infinitesimal level, see Remark 3.4 for details.

While the uniform metrics of type I could be viewed as the extremal case $d=0$ of $\mathcal{M}_{-1}^{d}$, the resulting compactifications of the moduli space are very different. For our class of metrics, the analogue of the Deligne-Mumford compactness theorem takes the following form.

Theorem 1.2 Let $M$ be a compact oriented surface of genus $\gamma$ with boundary curves $\Gamma_{1}, \ldots, \Gamma_{k}$ and negative Euler characteristic, let $\mathcal{N}_{-1}^{d}, d>0$, be the set of all hyperbolic metrics on $M$ for which (1.1) is satisfied and suppose that $g^{(j)}$ is a sequence in $\mathcal{M}_{-1}^{d}$ for which the lengths of the boundary curves are bounded above uniformly.

Then, after passing to a subsequence, $\left(M, g^{(j)}\right)$ converges to a complete hyperbolic surface $\left(\Sigma, g_{\infty}\right)$ with the same number of boundary curves, all satisfying (1.1), where $\Sigma$ is obtained from $M$ by removing a collection $\mathscr{E}=\left\{\sigma^{j}, j=1, \ldots, \kappa\right\}$ of $\kappa \in$ $\{0, \ldots, 3(\gamma-1)+2 k\}$ pairwise disjoint homotopically non-trivial simple closed curves in the interior of $M$ and the convergence is to be understood as follows:

For each $j$, there exists a collection $\mathscr{E}^{(j)}=\left\{\sigma_{i}^{(j)}, i=1, \ldots, \kappa\right\}$ of pairwise disjoint simple closed geodesics in $\left(M, g^{(j)}\right)$ of length $L_{g^{(j)}}\left(\sigma_{i}^{(j)}\right) \rightarrow 0$ as $j \rightarrow \infty$ and a diffeomorphism $f_{j}: \Sigma \rightarrow M \backslash \cup_{i=1}^{\kappa} \sigma_{i}^{(j)}$ such that

$$
f_{j}^{*} g^{(j)} \rightarrow g^{\infty} \text { smoothly locally on } \Sigma \text {. }
$$

The above results assure that the metrics are well controlled near the boundary even if the conformal structure degenerates, see also Remark 3.3, and that in particular no boundary curve can be 'lost'. Both of these properties are crucial in applications where $(M, g)$ plays the role of the domain of a PDE with prescribed boundary conditions, e.g. if one wants to extend ideas of Teichmüller harmonic map flow, introduced for maps from tori by Ding, Li and Liu in [9] and in the joint work [19] of Topping and the author for maps from general closed surfaces, to the setting of maps from general surfaces with boundary in order to flow to solutions of the Douglas-Plateau problem.

In particular, if one hopes to prove global existence results, as obtained for closed surfaces in [20] and [21] for Teichmüller harmonic map flow and in [5] for Ricci- 
harmonic map flow, for geometric flows on surfaces with boundary, it is important that the most delicate region for the PDE, i.e. the boundary region, and the most delicate region for the evolution of the domain metric, which for hyperbolic metrics are sets of small injectivity radius, do not overlap but are instead far apart as is the case for our class of metrics $\mathcal{M}_{-1}^{d}$.

This paper is organised as follows. In Sect. 2 we consider the problem of finding hyperbolic metrics in a given conformal class with prescribed positive geodesic curvatures $\left.k_{g}\right|_{\Gamma_{i}}=c_{i}$ and analyse the properties of such metrics. The main difficulty here lies in the fact that for $c_{i}>0$, the boundary condition has the wrong sign to apply known existence results as found, e.g. in [8] and the corresponding variational problems contain negative boundary terms that have to be analysed carefully. Based on the results and estimates proven in Sect. 2, we will then give the proofs of the main results in Sect. 3.

\section{Hyperbolic Surfaces with Boundary Curves of Prescribed Positive Curvature}

In this section we prove the existence and uniqueness of hyperbolic metrics for which the boundary curves have prescribed positive constant geodesic curvature and establish several key properties of these metrics, which will be the basis of the proofs of our main results given in Sect. 3. We show in particular.

Lemma 2.1 Let $M$ be an oriented surface with boundary $\partial M=\bigcup_{i=1}^{k} \Gamma_{i}$ with $\chi(M)=$ $2(1-\gamma)-k<0$ and let $\mathrm{c}$ be any conformal structure on $M$. Then for any $c=$ $\left(c_{1}, \ldots, c_{k}\right) \in[0,1)^{k}$, there exists a unique metric $g_{c}$ on $M$ compatible with $\mathrm{c}$ so that

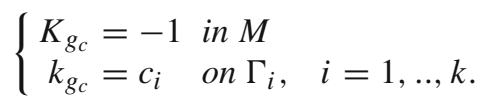

This result is of course true also for $c_{i}<0$, and in that case is indeed easier to prove as the boundary term in the corresponding variational integral has the right sign. We are, however, not interested in the properties of representatives with $c_{i} \leq 0$ as their boundary curves can collapse if the conformal structure degenerates, the very feature of the existing approaches of uniformisation that we want to avoid with our construction.

We recall that under a conformal change $g=e^{2 u} g_{0}$ the Gauss curvature transforms by

$$
K_{g}=e^{-2 u}\left(K_{g_{0}}-\Delta_{g_{0}} u\right)
$$

while, denoting by $n_{g_{0}}$ the outer unit normal of $\left(M, g_{0}\right)$, the geodesic curvature $k_{g}$ is characterised by

$$
\frac{\partial u}{\partial n_{g_{0}}}+k_{g_{0}}=k_{g} \cdot e^{u} .
$$

In the following we let $g_{0}$ be the unique metric so that $\left(M, g_{0}\right)$ is hyperbolic with geodesic boundary curves (which can, e.g. be obtained by doubling the surface and applying the classical uniformisation theorem), and write for short $n=n_{g_{0}}$. 
Thus $g=e^{2 u} g_{0}$ satisfies (2.1) if and only if

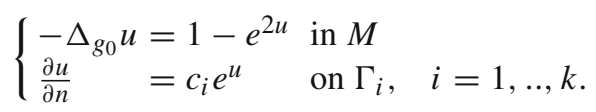

Lemma 2.1 is hence an immediate consequence of the following more refined result on solutions of the above PDE that we will prove in the present section.

Proposition 2.2 Let $\left(M, g_{0}\right)$ be an oriented hyperbolic surface with geodesic boundary curves $\Gamma_{1}, \ldots, \Gamma_{k}$. Then for any $c=\left(c_{1}, \ldots, c_{k}\right) \in[0,1)^{k}$ the equation (2.4) has a unique weak solution $u_{c} \in H^{1}\left(M, g_{0}\right)$ and this solution is smooth up to the boundary of $M$. In addition, the map

$$
[0,1)^{k} \ni c \mapsto u_{c} \in H^{1}\left(M, g_{0}\right)
$$

is of class $C^{1}$.

We begin by establishing the existence of solutions to (2.4) based on the direct method of calculus of variations. Solutions of (2.4) correspond to critical points of

$$
I_{c}(u)=\int_{M}|d u|_{g_{0}}^{2}+e^{2 u}-2 u d v_{g_{0}}-\sum_{i} 2 c_{i} \int_{\Gamma_{i}} e^{u} d S_{g_{0}},
$$

which is well defined on $H^{1}\left(M, g_{0}\right)$ as the Moser-Trudinger inequality [26] and its trace versions, see, e.g. [14], ensure in particular that for any $q<\infty$

$$
\sup _{u \in H^{1}\left(M, g_{0}\right),\|u\|_{H^{1}\left(M, g_{0}\right)} \leq 1} \int_{M} e^{q|u|} d v_{g_{0}}+\int_{\partial M} e^{q|u|} d S_{g_{0}}<\infty .
$$

A well-known consequence of this estimate is that for every $1<p<\infty$, the maps

$$
H^{1}\left(M, g_{0}\right) \ni u \mapsto e^{u} \in L^{p}\left(M, g_{0}\right) \text { and } H^{1}\left(M, g_{0}\right) \ni u \mapsto \operatorname{tr}_{\partial M}\left(e^{u}\right) \in L^{p}\left(\partial M, g_{0}\right)
$$

are compact operators: Any bounded sequence in $H^{1}$ has a subsequence which converges weakly in $H^{1}$, strongly in $L^{2}$ and whose traces converge strongly in $L^{2}$. The corresponding sequences $e^{u_{n}}$ and $\operatorname{tr}_{\partial M}\left(e^{u_{n}}\right)$ hence converge in measure and, thanks to (2.6) (applied, e.g. for $q=2 p$ ), are $p$-equiintegrable so converge strongly in $L^{p}$ by Vitali's convergence theorem.

An immediate consequence of the compactness of the operators in (2.7) is that $I_{C}$ is weakly lower semicontinuous on $H^{1}\left(M, g_{0}\right)$. Hence, to establish the existence of a minimiser of $I_{c}$ in $H^{1}\left(M, g_{0}\right)$, and thus a solution of (2.4), it suffices to prove that $I_{c}$ is also coercive on $H^{1}\left(M, g_{0}\right)$. To deal with the negative boundary terms, we will use that on hyperbolic surfaces with geodesic boundary curves, the trace theorem is valid in the following form, in particular with leading order term on the right-hand side appearing with a factor of 1 . 
Lemma 2.3 For any $\bar{L}<\infty$, there exists a constant $C_{1}=C_{1}(\bar{L})<\infty$ so that the estimate

$$
\int_{\partial M}|w| d S_{g_{0}} \leq \int_{M}|d w|_{g_{0}} d v_{g_{0}}+C_{1} \int_{M}|w| d v_{g_{0}}
$$

holds true for any oriented hyperbolic surface $\left(M, g_{0}\right)$ with geodesic boundary curves of length $L_{g_{0}}\left(\Gamma_{i}\right) \leq \bar{L}, i=1, \ldots, k$, and every $w \in W^{1,1}\left(M, g_{0}\right)$.

Proof of Lemma 2.3 We derive this estimate from the corresponding trace estimate

$$
\int_{\{0\} \times S^{1}}|w| d \theta \leq \int_{0}^{X} \int_{S^{1}}\left|\partial_{s} w\right| d \theta d s+X^{-1} \int_{0}^{X} \int_{S^{1}}|w| d \theta d s
$$

on Euclidean cylinders $[0, X] \times S^{1}$ and the properties of hyperbolic collars as follows. We first recall that the classical Collar lemma of Keen-Randol [18] yields the existence of pairwise disjoint neighbourhoods $\mathrm{C}\left(\Gamma_{i}\right)$ of the boundary curves which are isometric to the cylinders $\left(-X\left(\ell_{i}\right), 0\right] \times S^{1}$ equipped with $\rho_{\ell_{i}}(s)^{2}\left(d s^{2}+d \theta^{2}\right)$, where $\ell_{i}=$ $L_{g_{0}}\left(\Gamma_{i}\right)$ and where

$$
\rho_{\ell}(s)=\frac{\ell}{2 \pi}\left(\cos \left(\frac{\ell}{2 \pi} s\right)\right)^{-1} \text { and } X(\ell)=\frac{2 \pi}{\ell}\left(\frac{\pi}{2}-\arctan \left(\sinh \left(\frac{\ell}{2}\right)\right)\right),
$$

with the boundary curve $\Gamma_{i}$ corresponding to $\{0\} \times S^{1}$. We hence obtain from (2.9) that

$$
\begin{aligned}
\int_{\Gamma_{i}}|w| d S_{g_{0}}= & \rho_{\ell_{i}}(0) \int_{\{0\} \times S^{1}}|w| d \theta \leq \int_{0}^{X\left(\ell_{i}\right)} \int_{S^{1}} \rho_{\ell_{i}}^{-1}\left|\partial_{s} w\right| \rho_{\ell_{i}}^{2} d \theta d s \\
& +\frac{1}{X\left(\ell_{i}\right) \rho_{\ell_{i}}(0)} \int_{0}^{X\left(\ell_{i}\right)} \int_{S^{1}}|w| \rho_{\ell_{i}}^{2} d \theta d s \\
& \leq \int_{\mathcal{C}\left(\Gamma_{i}\right)}|d w|_{g_{0}} d v_{g_{0}}+c_{\bar{L}}^{-1} \int_{\mathcal{C}_{\left(\Gamma_{i}\right)}}|w| d v_{g_{0}},
\end{aligned}
$$

for every $i$, where we use that $\rho_{\ell}(s) \geq \rho_{\ell}(0) \geq \frac{c_{\bar{L}}}{X(\ell)}$ for some $c_{\bar{L}}>0$ and $\ell \in(0, \bar{L}]$. As the collars are disjoint, this implies the claim of the lemma.

Returning to the proof of the first part of Proposition 2.2, and hence of the coercivity of $I_{c}$ defined in (2.5), we now set $\bar{c}:=\max \left\{c_{i}\right\}<1$ and apply Lemma 2.3 to bound

$$
\sum_{i} c_{i} \int_{\Gamma_{i}} e^{u} d S_{g_{0}} \leq \bar{c} \int_{\partial M} e^{u} d S_{g_{0}} \leq \frac{1}{2} \bar{c} \int|d u|_{g_{0}}^{2}+e^{2 u} d v_{g_{0}}+C_{1} \int e^{u} d v_{g_{0}},
$$


where all integrals are computed over $M$ unless specified otherwise. Writing $-2 u=$ $2|u|-4 u^{+}$for $u^{+}=\max \{u, 0\}$, we can thus estimate

$$
\begin{aligned}
I_{c}(u) \geq & (1-\bar{c}) \int|d u|_{g_{0}}^{2}+e^{2 u} d v_{g_{0}}+2 \int|u| d v_{g_{0}}-4 \int u^{+} d v_{g_{0}}-2 C_{1} \int e^{u} d v_{g_{0}} \\
\geq & \frac{1}{2}(1-\bar{c}) \int|d u|_{g_{0}}^{2}+e^{2 u} d v_{g_{0}}+2 \int|u| d v_{g_{0}} \\
& +\int \frac{1}{2}(1-\bar{c}) e^{2 u^{+}}-4 u^{+}-2 C_{1} e^{u^{+}} d v_{g_{0}} \\
\geq & \frac{1}{2}(1-\bar{c}) \int|d u|_{g_{0}}^{2}+e^{2 u} d v_{g_{0}}+2 \int|u| d v_{g_{0}}-C
\end{aligned}
$$

for a constant $C$ that is allowed to depend on $\bar{c} \in[0,1), \chi(M)$, and hence $\operatorname{Area}(M, g)=-2 \pi \chi(M)$, and an upper bound $\bar{L}$ on the length of the boundary curves of $\left(M, g_{0}\right)$.

Coercivity of $I_{c}$ now easily follows: If $\left\|d u_{n}\right\|_{L^{2}\left(M, g_{0}\right)} \rightarrow \infty$ then clearly $I_{c}\left(u_{n}\right) \rightarrow$ $\infty$ while for sequences with $\left\|u_{n}\right\|_{H^{1}\left(M, g_{0}\right)} \rightarrow \infty$ and $\left\|d u_{n}\right\|_{L^{2}\left(M, g_{0}\right)} \leq C$, the Poincaré inequality implies that also $\left|f u_{n} d v_{g_{0}}\right| \rightarrow \infty$, so $I_{c}\left(u_{n}\right) \geq 2 \int\left|u_{n}\right| d v_{g_{0}}-$ $C \geq 2 \operatorname{Area}\left(M, g_{0}\right) \cdot\left|f u_{n} d v_{g_{0}}\right|-C \rightarrow \infty$.

This establishes the existence of a weak solution $u_{c} \in H^{1}\left(M, g_{0}\right)$ to (2.4) for any $c \in[0,1)^{k}$. Since the non-linearity in the Neumann problem (2.4) is subcritical, the regularity theorem $[8$, Théorème 1$]$ of Cherrier applies and yields that every weak solution of (2.4) is indeed smooth up to the boundary. At the same time, we remark that we could not have used the results of [8] to establish existence of solutions, as our boundary data have the wrong sign.

Remark 2.4 As we only use that the geodesic curvature $k_{g}$ is strictly less than 1 , the above proof indeed shows that for any given functions $k_{i} \in L^{p}\left(\Gamma_{i}\right), p>1$, for which $k_{i} \leq \bar{c}$ for some $\bar{c}<1$, there exists a hyperbolic metric $g$ compatible to $\mathrm{c}$ with $k_{g}=k_{i}$ on $\Gamma_{i}, i=1, \ldots, k$.

We will prove the other claims of Proposition 2.2 at the end of the section based on the properties of the surfaces $\left(M, g_{c}\right)$ that we discuss now, including the following version of the collar lemma.

Lemma 2.5 Let $(M, g)$ be an oriented hyperbolic surface with boundary curves of constant geodesic curvature $\left.k_{g}\right|_{\Gamma_{i}} \equiv c_{i} \in[0,1)$. Then for each $i \in\{1, \ldots, k\}$, there exists a unique simple closed geodesic $\gamma_{i}$ in $(M, g)$ that is homotopic to $\Gamma_{i}$ and there exist pairwise disjoint neighbourhoods $\mathrm{C}\left(\Gamma_{i}\right)$ of the boundary curves $\Gamma_{i}$ in $(M, g)$ which are isometric to cylinders

$$
\left(-X\left(\ell_{i}\right), Y\left(\ell_{i}, c_{i}\right)\right] \times S^{1} \text { with metric } \rho_{\ell_{i}}(s)^{2}\left(d s^{2}+d \theta^{2}\right)
$$

where $\rho_{\ell_{i}}$ and $X\left(\ell_{i}\right)$ are given by (2.10), $\ell_{i}=L_{g}\left(\gamma_{i}\right)$, and where

$$
Y\left(\ell_{i}, c_{i}\right)=\frac{2 \pi}{\ell_{i}} \arcsin \left(c_{i}\right)
$$

In these coordinates $\Gamma_{i}$ corresponds to $\left\{Y\left(\ell_{i}, c_{i}\right)\right\} \times S^{1}$ while $\gamma_{i}$ corresponds to $\{0\} \times S^{1}$. 
Proof We note that since our surface is hyperbolic, the Dirichlet energy of maps $u: S^{1} \rightarrow(M, g)$ has a unique minimiser in the homotopy class of $\Gamma_{i}$, c.f. [10], which coincides with $\Gamma_{i}$ if $c_{i}=0$. Otherwise, $\Gamma_{i}$ has positive geodesic curvature so the image of this minimiser must lie in the interior of $M$ and hence be the desired simple closed geodesic.

We let $\mathrm{C}^{+}\left(\Gamma_{i}\right)$ be the connected component of $\mathcal{M}_{-1} \backslash \bigcup_{i} \gamma_{i}$ that is bounded by $\gamma_{i}$ and $\Gamma_{i}$ and set $M_{0}:=M \backslash \bigcup_{i} \mathrm{e}^{+}\left(\Gamma_{i}\right)$. As $\left(M_{0}, g\right)$ is hyperbolic with geodesic boundary, the Collar lemma [18] gives disjoint neighbourhoods $\mathrm{C}^{-}\left(\gamma_{i}\right)$ of $\gamma_{i}$ in $M_{0}$ that are isometric to $\left(\left(-X\left(\ell_{i}\right), 0\right] \times S^{1}, \rho_{\ell_{i}}(s)\left(d s^{2}+d \theta^{2}\right)\right)$, with $\rho_{\ell}$ and $X(\ell)$ given by (2.10). The resulting disjoint neighbourhoods $\mathcal{C}\left(\Gamma_{i}\right):=\mathcal{C}^{-}\left(\gamma_{i}\right) \cup \mathcal{C}^{+}\left(\Gamma_{i}\right)$ of $\Gamma_{i}$ in our original surface $M$ are bounded by curves of constant geodesic curvature and are isometric to a subset of the complete hyperbolic cylinder $\left(\left(-\frac{\pi^{2}}{\ell_{i}}, \frac{\pi^{2}}{\ell_{i}}\right) \times S^{1}, \rho_{\ell_{i}}^{2}\left(d s^{2}+d \theta^{2}\right)\right)$ around a geodesic of length $\ell_{i}$, where such an isometry can, e.g. be obtained by using the fibration of $\mathcal{C}\left(\Gamma_{i}\right)$ by the geodesics that cross $\gamma_{i}$ orthogonally. We note that the only closed curves of constant geodesic curvature in such a cylinder are circles $\{s\} \times S^{1}$, whose curvature is

$$
k_{g}=\rho^{-1} \frac{\partial}{\partial s} \log (\rho(s))+k_{g_{\text {eucl }}}=\rho^{-2} \partial_{s} \rho=\sin \left(\frac{\ell}{2 \pi} s\right),
$$

compare (2.3); indeed, comparing the curvature of any other closed curve $\sigma$ with the one of the circles $\left\{s_{ \pm}\right\} \times S^{1}$ through points $P_{ \pm}=\left(s_{ \pm}, \theta_{ \pm}\right)$of $\sigma$ with extremal $s$ coordinate, we get

$$
k_{g}(\sigma)\left(P_{+}\right) \geq k_{g}\left(\left\{s_{+}\right\} \times S^{1}\right)=\sin \left(\frac{\ell}{2 \pi} s_{+}\right)>\sin \left(\frac{\ell}{2 \pi} s_{-}\right) \geq k_{g}(\sigma)\left(P_{-}\right) .
$$

The collar neighbourhood $\mathcal{C}\left(\Gamma_{i}\right)$ obtained above must hence be isometric to a cylinder $\left((-X, Y] \times S^{1}, \rho_{\ell_{i}}^{2}\left(d s^{2}+d \theta^{2}\right)\right)$ where, by (2.12), $X$ and $Y$ are as described in the lemma.

We also use the following standard property of Riemann surfaces.

Remark 2.6 For any given oriented Riemann surface $(M, \mathrm{c})$ with boundary curves $\Gamma_{1}, \ldots \Gamma_{k}$, there exists a number $\bar{Z}$ so that the following holds true. Let $U$ be any neighbourhood of one of the boundary curves $\Gamma_{i}$ which is conformal to a cylinder $(0, Z] \times S^{1}$. Then $Z \leq \bar{Z}$.

For the sake of completeness, we include a short proof of this well-known fact in the appendix. Combining it with Lemma 2.5, we get

Corollary 2.7 For any conformal structure c on $M$ there exists $\delta>0$ so that the following holds true. Let $g$ be any hyperbolic metric on $M$ for which $\left.k_{g}\right|_{\Gamma_{i}} \equiv c_{i} \in[0,1)$, $i=1 \ldots k$, and let $\gamma_{i}$ be the geodesics in $(M, g)$ that are homotopic to the boundary curves $\Gamma_{i}$. Then

$$
\ell_{i}:=L_{g_{c}}\left(\gamma_{i}\right) \geq \delta \text { and } L_{g_{c}}\left(\Gamma_{i}\right)=\frac{\ell_{i}}{\sqrt{1-c_{i}^{2}}} \geq \frac{\delta}{\sqrt{1-c_{i}^{2}}},
$$

in particular $L_{g_{c}}\left(\Gamma_{i}\right) \rightarrow \infty$ as $c_{i} \uparrow 1$. 
The bound on $\ell_{i}$ follows directly from Lemma 2.5 and Remark 2.6, applied for $Z=X\left(\ell_{i}\right) \rightarrow \infty$ as $\ell_{i} \rightarrow 0$, while the expression for $L_{g_{c}}\left(\Gamma_{i}\right)$ follows from (2.10) and (2.11).

For these surfaces, we can now prove the following version of the trace theorem.

Lemma 2.8 Let $(M, g)$ be an oriented hyperbolic surface with boundary curves of constant geodesic curvature $\left.k_{g}\right|_{\Gamma_{i}} \equiv c_{i} \in[0,1)$ and let $\mathrm{C}^{+}\left(\Gamma_{i}\right)$ be the subset of the collar $\mathcal{C}\left(\Gamma_{i}\right)$ described in Lemma 2.5 that is bounded by $\Gamma_{i}$ and the corresponding geodesic $\gamma_{i}$. Then

$$
c_{i} \int_{\Gamma_{i}}|w| d S_{g} \leq \int_{\mathrm{C}^{+}\left(\Gamma_{i}\right)}|w| d v_{g}+c_{i} \int_{\mathrm{C}^{+}\left(\Gamma_{i}\right)}|d w|_{g} d v_{g}
$$

holds true for any $w \in W^{1,1}(M, g)$. Furthermore, there exists $\varepsilon>0$, allowed to depend on both the lengths $\ell_{i}$ of the geodesics $\gamma_{i}$ and the curvatures $c_{i}$, so that for every $w \in W^{1,1}(M, g)$

$$
\int_{\partial M}\left(k_{g}+\varepsilon\right)|w| d S_{g} \leq(1-\varepsilon) \int_{M}|w| d v_{g}+(1-\varepsilon) \int_{M}|d w|_{g} d v_{g} .
$$

We note that the above lemma assures in particular that if $w \in H^{1}(M, g)$, then

$$
\int_{\partial M}\left(k_{g}+\varepsilon\right) w^{2} d S_{g} \leq(1-\varepsilon) \int_{M}|d w|_{g}^{2}+2 w^{2} d v_{g}
$$

Proof of Lemma 2.8 From Gauss-Bonnet and (2.12) we obtain that for $s \in\left[0, Y_{i}\right]$, $Y_{i}=Y\left(\ell_{i}, c_{i}\right)$

$$
\begin{aligned}
\int_{0}^{s} \rho_{\ell_{i}}^{2}(x) d x & =\frac{1}{2 \pi} \operatorname{Area}_{g}\left([0, s] \times S^{1}\right)=\frac{1}{2 \pi} \int_{\{s\} \times S^{1}} k_{g} d S_{g} \\
& =\left.\rho_{\ell_{i}}(s) k_{g}\right|_{\{s\} \times S^{1}}=\rho_{\ell_{i}}(s) \sin \left(\frac{\ell_{i}}{2 \pi} s\right),
\end{aligned}
$$

$(s, \theta)$ collar coordinates on $\mathrm{C}\left(\Gamma_{i}\right)$, in particular $\int_{0}^{Y_{i}} \rho_{\ell_{i}}^{2}=\rho_{\ell_{i}}\left(Y_{i}\right) \cdot c_{i}$. Multiplying

$$
\int_{\left\{Y_{i}\right\} \times S^{1}}|w| d \theta=\int_{\left\{s_{0}\right\} \times S^{1}}|w| d \theta+\int_{s_{0}}^{Y_{i}} \int_{S^{1}} \partial_{S}|w| d \theta d s
$$

with $\rho_{\ell_{i}}\left(s_{0}\right)^{2}$ and integrating over $s_{0} \in\left[0, Y_{i}\right]$ using Fubini hence gives the desired bound of

$$
\begin{aligned}
c_{i} \int_{\Gamma_{i}}|w| d S_{g} & =\int_{\mathcal{C}^{+}\left(\Gamma_{i}\right)}|w| d v_{g}+\int_{0}^{Y_{i}} \int_{S^{1}} \partial_{s}|w| \cdot \rho_{\ell_{i}}(s) \sin \left(\frac{\ell_{i}}{2 \pi} s\right) d \theta d s \\
& \leq \int_{\mathcal{C}^{+}\left(\Gamma_{i}\right)}|w| d v_{g}+c_{i} \cdot \int_{\mathcal{C}^{+}\left(\Gamma_{i}\right)}|d w|_{g} d v_{g} .
\end{aligned}
$$


Multiplying (2.17) with $\rho_{\ell_{i}}\left(Y_{i}\right)$ and averaging over $s_{0} \in\left(-X_{i}, 0\right], X_{i}=X\left(\ell_{i}\right)$, also yields

$$
\begin{aligned}
\int_{\Gamma_{i}}|w| d S_{g} & \leq \rho_{\ell_{i}}\left(Y_{i}\right) \cdot X_{i}^{-1} \int_{-X_{i}}^{0} \int_{S^{1}}|w| d \theta d s+\rho_{\ell_{i}}\left(Y_{i}\right) \int_{-X_{i}}^{Y_{i}} \int_{S^{1}}\left|\partial_{s} w\right| d \theta d s \\
& \leq C_{2} \int_{\mathcal{C}\left(\Gamma_{i}\right) \backslash \mathcal{C}^{+}\left(\Gamma_{i}\right)}|w| d v_{g}+C_{3} \int_{\mathcal{C}\left(\Gamma_{i}\right)}|d w|_{g} d v_{g}
\end{aligned}
$$

now for constants $C_{2,3}$ that depend both on $\ell_{i}=2 \pi \min \rho_{\ell_{i}}(\cdot)$ and $L_{g}\left(\Gamma_{i}\right)=$ $2 \pi \rho_{\ell_{i}}\left(Y_{i}\right)$. To obtain the second claim of the lemma, we now combine (2.18), multiplied by $(1-\varepsilon)$ for some $\varepsilon \in(0,1)$ chosen below, and (2.19), multiplied by $\left(1+c_{i}\right) \cdot \varepsilon$, to conclude that

$$
\begin{aligned}
\left(c_{i}+\varepsilon\right) \int_{\Gamma_{i}}|w| d S_{g} \leq & (1-\varepsilon) \int_{\mathcal{C}^{+}\left(\Gamma_{i}\right)}|w| d v_{g}+C_{2}\left(1+c_{i}\right) \cdot \varepsilon \int_{\mathcal{C}\left(\Gamma_{i}\right) \backslash \mathcal{C}^{+}\left(\Gamma_{i}\right)}|w| d v_{g} \\
& +\left[c_{i}(1-\varepsilon)+C_{3}\left(1+c_{i}\right) \cdot \varepsilon\right] \int_{\mathcal{C}\left(\Gamma_{i}\right)}|d w|_{g} d v_{g}
\end{aligned}
$$

For $\varepsilon>0$ chosen small enough to ensure that $2 C_{2} \varepsilon \leq 1-\varepsilon$ and $2 C_{3} \varepsilon \leq\left(1-c_{i}\right)(1-\varepsilon)$, this yields the second claim (2.15) of the lemma as the collar neighbourhoods are disjoint.

We are now in a position to prove the following a priori bounds for PDEs related to (2.4)

Lemma 2.9 Let $M$ be an oriented surface with boundary curves $\Gamma_{1}, \ldots, \Gamma_{k}$ and let $g$ be a metric on $M$ which satisfies (2.1) for some $c \in[0,1)^{k}$.

Then there exist constants $C_{4,5}$, allowed to depend both on $c$ and the underlying conformal structure, so that the following holds true for any $f \in L^{2}(M, g)$ and $h \in L^{2}(\partial M, g)$.

(i) Suppose that $w \in H^{1}(M, g)$ is a weak solution of

$$
-\Delta_{g} w=1-e^{2 w}+f \quad \text { in } M \quad \text { with } \quad \frac{\partial w}{\partial n_{g}}=k_{g}\left(e^{w}-1\right)+h \text { on } \partial M
$$

for which furthermore $e^{w} \in H^{1}(M, g)$. Then

$$
\begin{aligned}
& \int_{M}|d u|_{g}^{2} e^{w}+\left(e^{w}-1\right)^{2}\left(e^{w}+1\right) d v_{g}+\int_{\partial M}\left(e^{w}-1\right)^{2} d S_{g} \\
& \quad \leq C_{4}\left(\|f\|_{L^{2}(M, g)}^{2}+\|h\|_{L^{2}(\partial M, g)}^{2}\right) .
\end{aligned}
$$

(ii) There exists a unique solution $v \in H^{1}(M, g)$ of the linearised problem

$$
-\Delta_{g} v+2 v=f \quad \text { in } M \quad \text { with } \quad \frac{\partial v}{\partial n_{g}}=k_{g} v+h \text { on } \partial
$$


and we have that

$$
\|v\|_{H^{1}(M, g)}^{2} \leq C_{5}\left(\|f\|_{L^{2}(M, g)}^{2}+\|h\|_{L^{2}(\partial M, g)}^{2}\right)
$$

Proof of Lemma 2.9 Let $w$ be as in the first part of the lemma and let $\varepsilon>0$ be as in Lemma 2.8. Testing (2.20) with $e^{w}-1 \in H^{1}(M, g)$, we may estimate

$$
\begin{aligned}
I:= & \int|d w|_{g}^{2} e^{w}+\left(e^{w}-1\right)^{2}\left(e^{w}+1\right) d v_{g}=\int_{\partial M} \frac{\partial w}{\partial n_{g}}\left(e^{w}-1\right) d S_{g} \\
& +\int f \cdot\left(e^{w}-1\right) d v_{g} \\
= & \int_{\partial M} k_{g}\left(e^{w}-1\right)^{2}+h\left(e^{w}-1\right) d S_{g}+\int f \cdot\left(e^{w}-1\right) d v_{g} \\
\leq & \int_{\partial M}\left(k_{g}+\varepsilon\right)\left(e^{w}-1\right)^{2} d S_{g}-\frac{\varepsilon}{2} \int_{\partial M}\left(e^{w}-1\right)^{2} d S_{g}+\frac{\varepsilon}{2} I \\
& +\frac{1}{2 \varepsilon}\left[\|h\|_{L^{2}(\partial M, g)}^{2}+\|f\|_{L^{2}(M, g)}^{2}\right] .
\end{aligned}
$$

By (2.15), the first term on the right is bounded by $(1-\varepsilon) \int\left(1-e^{w}\right)^{2}+2 e^{w}|d w|_{g} \mid 1-$ $e^{w} \mid d v_{g} \leq(1-\varepsilon) I$, so the first claim (2.21) of the lemma immediately follows.

To prove the second part of the lemma, we use that the variational integral

$$
F_{f, h}(v):=\int|d v|_{g}^{2}+2 v^{2}+2 f v d v_{g}-\int_{\partial M} k_{g} v^{2}+2 v h d S_{g}
$$

associated with (2.22) is coercive, as (2.16) implies

$$
\begin{aligned}
F_{f, h}(v) \geq & \varepsilon \int|d v|_{g}^{2}+2 v^{2} d v_{g}-2\|f\|_{L^{2}(M, g)} \cdot\|v\|_{L^{2}(M, g)} \\
& -\|h\|_{L^{2}(\partial M, g)}\|v\|_{L^{2}(\partial M, g)} \\
\geq & \varepsilon\|v\|_{H^{1}(M, g)}^{2}-\left(2\|f\|_{L^{2}(M, g)}+C\|h\|_{L^{2}(\partial M, g)}\right)\|v\|_{H^{1}(M, g)}
\end{aligned}
$$

Hence $F_{f, h}$ has a minimiser $v$ which is of course a solution of (2.22), and satisfies $F_{f, h}(v) \leq F_{f, h}(0)=0$ which, combined with (2.24), furthermore yields the claimed a priori estimate (2.23). Finally, this solution of (2.22) is unique as the difference $v$ of two solutions of (2.22) satisfies

$$
0=\int|d v|_{g}^{2}+2 v^{2} d v_{g}-\int_{\partial M} k_{g} v^{2} d S_{g} \geq \varepsilon\|v\|_{H^{1}(M, g)}^{2},
$$

again by (2.16), and must thus vanish.

As a next step towards completing the proof of Proposition 2.2, we show 
Lemma 2.10 Let $M$ be as in Lemma 2.9 and let $g$ be any metric for which (2.1) holds true for some $c \in[0,1)^{k}$. Then there exist numbers $0<\varepsilon_{0}<1-\max c_{i}$ and $C_{6}<\infty$ so that for any $b \in\left[-\varepsilon_{0}, \varepsilon_{0}\right]^{k}$ and any hyperbolic metric $\tilde{g}=e^{2 w} g$ with $\left.k_{\tilde{g}}\right|_{\Gamma_{i}}=c_{i}+b_{i}, i=1, \ldots, k$, we have

$$
\int_{M}\left(e^{w}+1\right)\left(e^{w}-1\right)^{2}+e^{w}|d w|_{g}^{2} d v_{g}+\|w\|_{H^{1}(M, g)}^{2} \leq C_{6} \max \left|b_{i}\right|^{2}
$$

In particular, the solution of (2.1) is unique.

Proof Let $b \in\left[-\varepsilon_{0}, \varepsilon_{0}\right]^{k}$, where $\varepsilon_{0}>0$ is determined later, set $\bar{b}=\max \left|b_{i}\right|$ and suppose that $\tilde{g}=e^{2 w} g$ is as in the lemma. From (2.2) and (2.3), we obtain that $w$ solves

$$
-\Delta_{g} w=1-e^{2 w} \quad \text { in } M \quad \text { with } \quad \frac{\partial w}{\partial n_{g}}=k_{g}\left(e^{w}-1\right)+b_{i} e^{w} \text { on } \Gamma_{i},
$$

i.e. satisfies (2.20) for $f \equiv 0$ and $\left.h\right|_{\Gamma_{i}}=b_{i} e^{w}$. We note that $e^{w} \in H^{1}(M, g)$, as we may characterise $w=u_{c+b}-u_{c}$ as difference of smooth solutions of (2.1), so we may bound $I:=\int_{M}|d w|_{g}^{2} e^{w}+\left(e^{w}+1\right)\left(e^{w}-1\right)^{2} d v_{g}+\int_{\partial M}\left(e^{w}-1\right)^{2} d S_{g}$ using the first part of Lemma 2.9 by

$$
I \leq C_{4}\left\|b e^{w}\right\|_{L^{2}(\partial M, g)}^{2} \leq 2 C_{4} \bar{b}^{2} L_{g}(\partial M)+2 C_{4} \bar{b}^{2} \int_{\partial M}\left(e^{w}-1\right)^{2} d S_{g} \leq C \bar{b}^{2}+C \varepsilon_{0}^{2} I .
$$

For $\varepsilon_{0}>0$ sufficiently small, this gives the bound $I \leq C \bar{b}^{2}$ on $I$ claimed in the lemma and it remains to establish the analogue bound on the $H^{1}$ norm of $w$. We first note that

$$
\left\|e^{w / 2}-1\right\|_{H^{1}(M)}^{2}=\int \frac{1}{4}|d w|_{g}^{2} e^{w}+\left(e^{w / 2}-1\right)^{2} d v_{g} \leq I \leq C \bar{b}^{2},
$$

where norms are computed with respect to $g$ and integrals over $M$ unless indicated otherwise. In particular $\left\|e^{w / 2}\right\|_{H^{1}(M)} \leq C$, and so of course $\left\|e^{3 w / 2}\right\|_{L^{4}(M)}+$ $\left\|e^{w}\right\|_{L^{2}(\partial M)} \leq C$, where all constants are allowed to depend on $(M, g)$ but not on $b$. Writing (2.26) in the form

$$
\begin{aligned}
-\Delta_{g} w & =\left(1-e^{w / 2}\right) \cdot\left(1+e^{w / 2}+e^{w}+e^{3 w / 2}\right) \text { on } M, \\
\frac{\partial w}{\partial n_{g}} & =c_{i}\left(e^{w / 2}-1\right)\left(e^{w / 2}+1\right)+b_{i} e^{w} \text { on } \Gamma_{i},
\end{aligned}
$$

and testing this equation with $w-\bar{w}_{M}, \bar{w}_{M}:=f_{M} w d v_{g}$, thus allows us to bound

$$
\begin{aligned}
\|d w\|_{L^{2}(M)}^{2} \leq & C\left\|w-\bar{w}_{M}\right\|_{L^{2}(M)}\left\|1-e^{w / 2}\right\|_{L^{4}(M)} \cdot\left(1+\left\|e^{3 u / 2}\right\|_{L^{4}(M)}\right) \\
& +\left\|w-\bar{w}_{M}\right\|_{L^{2}(\partial M)} \cdot\left[\left\|e^{w / 2}-1\right\|_{L^{4}(\partial M)} \cdot\left\|e^{w / 2}+1\right\|_{L^{4}(\partial M)}\right. \\
& \left.+\bar{b}\left\|e^{w}\right\|_{L^{2}(\partial M)}\right] \\
\leq & C\left\|w-\bar{w}_{M}\right\|_{H^{1}(M)} \cdot\left[\left\|e^{w / 2}-1\right\|_{H^{1}(M)}+\bar{b}\right] \leq C \bar{b} \cdot\|d w\|_{L^{2}(M)} .
\end{aligned}
$$


Having thus shown that $\|d w\|_{L^{2}(M)} \leq C \bar{b}$, it now remains to show that also $\left|\bar{w}_{M}\right| \leq C \bar{b}$, which of course follows if we prove that $\int|w| d v_{g} \leq C \bar{b}$. As $|x| \leq 2 e^{-\min (x / 2,0)}\left|e^{x / 2}-1\right|, x \in \mathbb{R}$, we already obtain from (2.27) that

$$
\int_{\{w>-4\}}|w| d v_{g} \leq 2 e^{2} \int_{M}\left|e^{w / 2}-1\right| d v_{g} \leq C \bar{b},
$$

so it remains to bound the corresponding integral over $\{w<-4\}$. To this end we note that as $\left\|e^{w / 2}\right\|_{L^{4}(M)} \leq C$, we obtain from (2.27) that, after reducing $\varepsilon_{0}$ if necessary,

$$
\int\left(e^{w}-1\right)^{2} d v_{g} \leq C\left\|e^{w / 2}-1\right\|_{H^{1}(M)}^{2} \leq C \bar{b}^{2} \leq C \varepsilon_{0}^{2} \leq \frac{1}{2} \operatorname{Area}(M)
$$

so $\operatorname{Area}_{g}(\{w<-3\}) \leq \operatorname{Area}_{g}\left(\left\{\left(e^{w}-1\right)^{2}>\frac{3}{4}\right\}\right) \leq \frac{4}{3} \int\left(e^{w}-1\right)^{2} d v_{g} \leq \frac{2}{3} \operatorname{Area}_{g}(M)$. Hence $v=(w+3)_{-}=\max (-(w+3), 0)$ vanishes on a set of measure at least $\alpha=\frac{1}{3} \operatorname{Area}_{g}(M)$, so the variant of the Poincaré inequality

$$
\|v\|_{L^{2}(M)} \leq C\|d v\|_{L^{2}(M)}, \quad C=C(\alpha,(M, g))
$$

valid for such functions $v$ implies that also

$$
\int_{\{w<-4\}}|w| d v_{g} \leq 4 \int_{\{w<-4\}} v d v_{g} \leq C\|d v\|_{L^{2}} \leq C\|d w\|_{L^{2}} \leq C \bar{b},
$$

which completes the proof of the lemma.

Lemmas 2.9 and 2.10 represent the main steps in the proof of the remaining claims of Proposition 2.2, which now follow by the following standard argument.

Let $S: c \mapsto u_{c} \in H^{1}\left(M, g_{0}\right)$ be the map that assigns to each $c \in[0,1)^{k}$ the unique solution $u_{c}$ of (2.4). We claim that $S$ is $C^{1}$ with $d S(c)(b)=v_{c, b}$, for $v_{c, b}$ the unique solutions of

$$
-\Delta_{g_{c}} v+2 v=0 \text { in } M, \quad \text { with } \quad \frac{\partial v}{\partial n_{g_{c}}}=c_{i} v+b_{i} \text { on } \Gamma_{i}, i \in\{1, \ldots, k\} .
$$

Here and in the following $g_{c}=e^{2 u_{c}} g_{0}$ is the unique metric satisfying (2.1).

Given $c \in[0,1)^{k}, b \in \mathbb{R}^{k}$, say with $|b|=1$, and $|\varepsilon| \leq 1-\max c_{i}$, we let $c_{\varepsilon}=c+\varepsilon b$ and set $w_{\varepsilon}:=S\left(c_{\varepsilon}\right)-S(c)$. As $g_{c_{\varepsilon}}=e^{2 S\left(c_{\varepsilon}\right)} g_{0}=e^{2 w_{\varepsilon}} g_{c}$ is hyperbolic with $k_{g_{\varepsilon}}=c_{\varepsilon}$, we have

$$
-\Delta_{g_{c}} w_{\varepsilon}+e^{2 w_{\varepsilon}}-1=0 \text { in } M \quad \text { with } \quad \frac{\partial w_{\varepsilon}}{\partial n_{g_{c}}}=\left(c_{i}+\varepsilon b_{i}\right) e^{w_{\varepsilon}}-c_{i} \text { on } \Gamma_{i},
$$

compare (2.3). Hence $\beta_{\varepsilon}:=S\left(c_{\varepsilon}\right)-S(c)-\varepsilon v_{c, b}=w_{\varepsilon}-\varepsilon v_{c, b}$ solves (2.22) for $g=g_{c}, f=1+2 w_{\varepsilon}-e^{2 w_{\varepsilon}}$ and $\left.h\right|_{\Gamma_{i}}=c_{i}\left(e^{w_{\varepsilon}}-\left(1+w_{\varepsilon}\right)\right)+\varepsilon b_{i}\left(e^{w_{\varepsilon}}-1\right)$, so for functions with 


$$
\begin{aligned}
|f| \leq 2 e^{2\left(w_{\varepsilon}\right)_{+}} \cdot\left(w_{\varepsilon}\right)^{2} & \leq 2\left(1+e^{2 w_{\varepsilon}}\right) \cdot w_{\varepsilon}^{2} \text { and }|h| \leq \frac{1}{2} e^{\left(w_{\varepsilon}\right)_{+}} w_{\varepsilon}^{2}+\varepsilon e^{\left(w_{\varepsilon}\right)_{+}}\left|w_{\varepsilon}\right| \\
& \leq\left(1+e^{w_{\varepsilon}}\right) \cdot\left[w_{\varepsilon}^{2}+\frac{1}{2} \varepsilon^{2}\right] .
\end{aligned}
$$

We recall from Lemma 2.10 that the $H^{1}$ norms of $e^{w_{\varepsilon}},|\varepsilon| \leq \varepsilon_{0}$, are uniformly bounded, and hence so are $\left\|e^{2 w_{\varepsilon}}\right\|_{L^{4}(M)}$ and $\left\|e^{w_{\varepsilon}}\right\|_{L^{4}(\partial M)}$. Using (2.23) as well as that $w_{\varepsilon}=\beta_{\varepsilon}+\varepsilon v_{c, b}$ we thus get

$$
\begin{aligned}
\left\|\beta_{\varepsilon}\right\|_{H^{1}\left(M, g_{c}\right)} \leq & C\left(\|f\|_{L^{2}\left(M, g_{c}\right)}+\|h\|_{L^{2}\left(\partial M, g_{c}\right)}\right) \leq C\left\|w_{\varepsilon}^{2}\right\|_{L^{4}\left(M, g_{c}\right)} \\
& +C\left\|w_{\varepsilon}^{2}\right\|_{L^{4}\left(\partial M, g_{c}\right)}+C \varepsilon^{2} \leq C\left\|\beta_{\varepsilon}\right\|_{H^{1}\left(M, g_{c}\right)}^{2} \\
& +C \varepsilon^{2}\left(1+\left\|v_{c, b}\right\|_{H^{1}\left(M, g_{c}\right)}^{2}\right) \leq C\left\|\beta_{\varepsilon}\right\|_{H^{1}\left(M, g_{c}\right)}^{2}+C \varepsilon^{2},
\end{aligned}
$$

where we use in the last step that (2.23) yields a bound on the norm of $v_{c, b}$ that is independent of $b$. As we know a priori that $\left\|\beta_{\varepsilon}\right\|_{H^{1}\left(M, g_{c}\right)} \leq\left\|w_{\varepsilon}\right\|_{H^{1}\left(M, g_{c}\right)}+$ $\varepsilon\left\|v_{c, b}\right\|_{H^{1}\left(M, g_{c}\right)} \leq C \varepsilon$, compare Lemma 2.10, we thus conclude that $\left\|\beta_{\varepsilon}\right\|_{H^{1}\left(M, g_{c}\right)} \leq$ $C \varepsilon^{2}$ and thus that $S$ is indeed Fréchet differentiable in $c$ with $d f(c)(b)=v_{c, b}$ as claimed.

We finally remark that $v_{c, b}$ depends continuously on $c$ as can be readily seen by using that $g_{\tilde{c}}=e^{2(S(\tilde{c})-S(c))}$ to view $v_{\tilde{c}, b}$ as solution of (2.22) for $g=g_{c}, f=$ $2 v\left(1-e^{2(S(\tilde{c})-S(c))}\right)$ and $\left.h\right|_{\Gamma_{i}}=b_{i}+\left(e^{S(\tilde{c})-S(c)}-1\right)\left(c_{i} v+b_{i}\right)+e^{S(\tilde{c})-S(c)}\left(\tilde{c}_{i}-c_{i}\right) v$ and applying Lemmas 2.9 and 2.10.

\section{Proof of the Main Results}

Based on the results of Sect. 2, we can now show the first part of Theorem 1.1 by proving

Lemma 3.1 Let $(M, \mathrm{c})$ be a compact oriented Riemann surface with boundary curves $\Gamma_{1}, \ldots, \Gamma_{k}$ and denote by $g_{c}, c \in[0,1)^{k}$, the unique metric compatible to $\mathrm{c}$ for which (2.1) holds. Then the map $f: c \mapsto\left(c_{i} \cdot L_{g_{c}}\left(\Gamma_{i}\right)\right)_{i}$ is a diffeomorphism from $(0,1)^{k}$ to $\left(\mathbb{R}^{+}\right)^{k}$. In particular, for every $d>0$ there exists a unique hyperbolic metric $g$ that is compatible with $\mathrm{C}$ and that satisfies (1.1).

Proof We first remark that $f: c \mapsto\left(c_{i} \cdot L_{g_{c}}\left(\Gamma_{i}\right)\right)_{i}$ is a $C^{1}$ map from $[0,1)^{k}$ to $\left(\mathbb{R}_{0}^{+}\right)^{k}$ as Proposition 2.2 establishes that $c \mapsto u_{c}$ is $C^{1}$ into $H^{1}$, while the trace version of the Moser-Trudinger inequality implies that $H^{1}\left(M, g_{0}\right) \ni u \mapsto \int_{\Gamma_{i}} e^{u} d S_{g_{0}}=L_{e^{2 u} g_{0}}\left(\Gamma_{i}\right)$ is $C^{1}$.

We now claim that $f:(0,1)^{k} \rightarrow\left(\mathbb{R}^{+}\right)^{k}$ is proper: To see this we first recall that Corollary 2.7 assures that $L_{g_{c}}\left(\Gamma_{i}\right) \rightarrow \infty$ as $c_{i} \rightarrow 1$ and hence that the preimage $f^{-1}(K)$ of any compact set $K \subset\left(\mathbb{R}_{0}^{+}\right)^{k}$ is a compact set in $[0,1)^{k}$. As $c \mapsto L_{g_{c}}\left(\Gamma_{i}\right)$ is continuous on $[0,1)^{k}$ we furthermore have a uniform upper bound on each $L_{g_{c}}\left(\Gamma_{i}\right)$ for $c \in f^{-1}(K)$. For compact subsets $K$ of $\left(\mathbb{R}^{+}\right)^{k}$ we hence obtain that the components $c_{i}$ of $c \in f^{-1}(K)$ are bounded away from zero uniformly and hence that $f^{-1}(K)$ is a compact subset of $(0,1)^{k}$ as required. 
By Hadamard's global inverse function theorem, see, e.g. [12, Chap. 6], the lemma thus follows provided we show that

$$
\operatorname{det}(d f(c)) \neq 0 \text { for every } c \in(0,1)^{k} \text {. }
$$

So suppose that there exists $c \in(0,1)^{k}$ so that $\operatorname{det}(d f(c))=0$. Hence there must be some non-trivial element $b$ of the kernel of $d f(c)$, i.e. $b \in \mathbb{R}^{k} \backslash\{0\}$ so that for every $i=1, \ldots, k$

$$
0=d f(c)(b)_{i}=b_{i} L_{g_{c}}\left(\Gamma_{i}\right)+\left.c_{i} \int_{\Gamma_{i}} \frac{d}{d \varepsilon}\right|_{\varepsilon=0} e^{u_{c}+\varepsilon v_{c, b}} d S_{g_{0}}=b_{i} L_{g_{c}}\left(\Gamma_{i}\right)+c_{i} \int_{\Gamma_{i}} v_{c, b} d S_{g_{c}},
$$

where $v_{c, b}=d S(c)(b)$ is characterised by (2.28). Testing (2.28) with $v_{c, b}$ and applying the trace estimate (2.16) of Lemma 2.8 , however, yields that

$$
\begin{aligned}
\int_{M}\left|d v_{c, b}\right|_{g_{c}}^{2}+2 v_{c, b}^{2} d v_{g_{c}} & =\int_{\partial M} k_{g_{c}} v_{c, b}^{2} d S_{g_{c}}+\sum_{i} b_{i} \int_{\Gamma_{i}} v_{c, b} d S_{g_{c}} \\
& \leq(1-\varepsilon) \int_{M}\left|d v_{c, b}\right|_{g_{c}}^{2}+2 v^{2} d v_{g_{c}}+\sum_{i} b_{i} \int_{\Gamma_{i}} v_{c, b} d S_{g_{c}}
\end{aligned}
$$

Since $v_{c, b}$ cannot vanish identically as $b \neq 0$, there hence must be at least one $i \in$ $\{1, \ldots k\}$ with

$$
\operatorname{sign}\left(b_{i}\right) \int_{\Gamma_{i}} v_{c, b} d S_{g_{c}}>0
$$

which contradicts (3.1) as $c_{i}>0$.

Having thus proven that each conformal class is represented by a unique metric $g \in \mathcal{M}_{-1}^{d}$, we now obtain the remaining claims of Theorem 1.1 from the following lemma which is based on Lemma 2.5 and Corollary 2.7.

Lemma 3.2 Let $M$ be as in Theorem 1.1 and let $g \in \mathcal{M}_{-1}^{d}, d>0$. Then there is a unique geodesic $\gamma_{i}$ in $(M, g)$ homotopic to the boundary curve $\Gamma_{i}$, its length $\ell_{i}$ is related to the length of $\Gamma_{i}$ by (1.2) and $\Gamma_{i}$ is surrounded by a collar neighbourhood that is isometric to

$$
\left(\left(-X\left(\ell_{i}\right), \bar{X}_{d}\left(\ell_{i}\right)\right] \times S^{1}, \rho_{\ell_{i}}\left(d s^{2}+d \theta^{2}\right)\right) \text { where } \bar{X}_{d}(\ell)=\frac{2 \pi}{\ell}\left(\frac{\pi}{2}-\arctan \left(\frac{\ell}{d}\right)\right)
$$

while $X(\ell)$ and $\rho_{\ell}$ are as in (2.10).

Proof The existence of such a geodesic was proven in Lemma 2.5 and the relation between $\ell_{i}=L_{g}\left(\gamma_{i}\right)$ and $L_{i}=L_{g}\left(\Gamma_{i}\right)$ follows from Corollary 2.7 which implies that $\ell_{i}^{2}=\left(1-\left(\left.k_{g}\right|_{\Gamma_{i}}\right)^{2}\right) L_{i}^{2}=L_{i}^{2}-d^{2}$. From Lemma 2.5 we then obtain that the boundary curve is surrounded by a collar as described in the above lemma where we know that 
$\bar{X}_{d}$ must be so that $\left.k_{g}\right|_{\Gamma_{i}}=\sin \left(\frac{\ell}{2 \pi} \bar{X}_{d}\left(\ell_{i}\right)\right)$. Combined with (2.13) this yields the condition $\frac{d}{\ell_{i}}=\frac{\left.k_{g}\right|_{\Gamma_{i}} L_{i}}{\sqrt{1-\left(\left.k_{g}\right|_{\Gamma_{i}}\right)^{2}} L_{i}}=\tan \left(\frac{\ell}{2 \pi} \bar{X}_{d}\left(\ell_{i}\right)\right)$, so $\bar{X}_{d}\left(\ell_{i}\right)$ must be given by (3.2).

Remark 3.3 We remark that while $X(\ell)$ and $\bar{X}_{d}(\ell)$ have a similar asymptotic behaviour as $\ell \rightarrow 0$ the behaviour of $X(\ell)$ and $\bar{X}_{d}(\ell)$ as $\ell \rightarrow \infty$ is very different, with $X(\ell)$ decaying exponentially, $X(\ell) \leq C \ell^{-1} e^{-\ell / 2}$, while $\bar{X}_{d}(\ell)$ is of order $\ell^{-2}$ for large $\ell$. This difference is significant due to its effect on the Teichmüller space and its completion with respect to the corresponding Weil-Petersson metric, which will be discussed in more detail in future work. To illustrate this, we note that for the corresponding metrics $G_{\ell}$ on cylinders (which were considered in [23] and are isometric to $\left(\left[-\bar{X}_{d}(\ell), \bar{X}_{d}(\ell)\right] \times S^{1}, \rho^{2}\left(d s^{2}+d \theta^{2}\right)\right)$ the change of the length of the central geodesic is controlled by $\frac{d \ell}{d t} \leq C \ell\left\|\partial_{t} G_{\ell(t)}\right\|_{L^{2}}$ if $\ell$ is large, which excludes the possibility that $\ell \rightarrow \infty$ along a curve of metrics of finite $L^{2}$-length. In this case we thus know that while the completion of the Teichmüller space includes the punctured limit obtained as $\ell \rightarrow 0$, it does not include any limiting object corresponding to $\ell$ becoming unbounded.

Remark 3.4 We note that the quantity $L_{g}\left(\Gamma_{i}\right)^{2}-L_{g}\left(\gamma_{i}\right)^{2}$ appears naturally also for horizontal curves of hyperbolic metrics on closed surfaces, as considered in [22]. Such curves move orthogonally to the action of diffeomorphisms and hence satisfy $\partial_{t} \hat{g}=\operatorname{Re}(\Omega)$ for holomorphic quadratic differentials $\Omega$ on $(M, \hat{g})$. Given a simple closed geodesic $\gamma \subset(M, \hat{g})$ and a closed curve $\Gamma \subset(\mathcal{C}(\gamma), \hat{g})$ with constant geodesic curvature, which is hence described by some $\{s\} \times S^{1}$ in collar coordinates, we can use the Fourier expansion of $\Omega=\sum_{j \in \mathbb{Z}} b_{j} e^{j(s+\mathrm{i} \theta)} d z^{2}$ to obtain that

$$
\begin{aligned}
\frac{d}{d t}\left[L_{\hat{g}(t)}(\Gamma)^{2}-L_{\hat{g}(t)}(\gamma)^{2}\right] & =L_{\hat{g}}(\Gamma) \int_{\{s\} \times S^{1}} \frac{\left(\partial_{t} \hat{g}\right)_{\theta \theta}}{\sqrt{\hat{g}_{\theta \theta}}} d \theta-L_{\hat{g}}(\gamma) \int_{\{s\} \times S^{1}} \frac{\left(\partial_{t} \hat{g}\right)_{\theta \theta}}{\sqrt{\hat{g}_{\theta \theta}}} d \theta \\
& =-2 \pi \operatorname{Re}\left(\sum_{j} b_{j}\left(e^{j s}-1\right) \int_{S^{1}} e^{i j \theta} d \theta\right)=0 .
\end{aligned}
$$

We finally give the proof of the analogue of the Deligne-Mumford compactness result for our class of metrics that we stated in Theorem 1.2. This proof is based on the proof of the corresponding result for surfaces with geodesic boundary curves as carried out in [11, Sect. IV.5]. For part of this proof, it will be more convenient to work with so-called Fermi coordinates $(x, \theta)$ instead of collar coordinates $(s, \theta)$ on a collar $\mathcal{C}(\sigma)$ around a simple closed geodesic $\sigma$. As indicated, the angular components of these two different sets of coordinates agree, while the $x$ coordinate of a point $p \in \mathcal{C}(\sigma)$ is given as the signed distance $x=x(s)=\operatorname{dist}_{g}(p, \sigma)$ to $\sigma$.

Proof of Theorem 1.2 For $\left(M, g^{(j)}\right)$ as in the theorem we denote by $\gamma_{i}^{(j)}, i=1, \ldots, k$, the (unique) geodesics in $\left(M, g^{(j)}\right)$ that are homotopic to $\Gamma_{i}$, and note that their lengths are bounded from above by $\ell_{i}^{(j)}:=L_{g^{(j)}}\left(\gamma_{i}^{(j)}\right) \leq L_{g^{(j)}}\left(\Gamma_{i}\right) \leq C$. We can thus pass to subsequence so that $\ell_{i}^{(j)} \rightarrow \ell_{i}^{\infty}$ as $j \rightarrow \infty$ for each $i=1, \ldots, k$, where, after 
relabelling, we may assume that $\ell_{i}^{\infty}=0$ for $1 \leq i \leq \kappa_{1}$ while $\ell_{i}>0$ for $\kappa_{1}+1 \leq i \leq k$ for some $\kappa_{1} \in\{0, \ldots, k\}$. As above we let $\mathcal{C}^{+}\left(\Gamma_{i}, g^{(j)}\right), i=1, \ldots, k$, be the halfcollars that are bounded by $\Gamma_{i}$ and the corresponding geodesic $\gamma_{i}^{(j)} \subset\left(M, g^{(j)}\right)$ and set $M^{(j)}:=M \backslash \bigcup_{i} \mathrm{e}^{+}\left(\Gamma_{i}^{(j)}\right)$.

As $\left(M^{(j)}, g^{(j)}\right)$ is a sequence of hyperbolic surfaces with geodesic boundary, we can apply the version of the Deligne-Mumford compactness theorem as found in [11, Prop. 5.1], or alternatively first double the surface and then apply the version of Deligne-Mumford for closed surfaces that is recalled, e.g. in [20, Prop. A.3]. After passing to a subsequence we thus obtain a collection $\mathscr{E}(j)=\left\{\sigma_{i}^{(j)}, i=1, \ldots, \kappa_{2}\right\}$, $\kappa_{2} \in\{0, \ldots, 3(\gamma-1)+k\}$, of simple closed geodesics in the interior of $\left(M^{(j)}, g^{(j)}\right)$ whose lengths tend to zero, so that for the surfaces $\Sigma^{(j)}=M^{(j)} \backslash\left(\bigcup_{i=1}^{\kappa_{2}} \sigma_{i}^{(j)} \cup\right.$ $\bigcup_{i=1}^{\kappa_{1}} \gamma_{i}^{(j)}$, which have with $\kappa_{1}+2 \kappa_{2}$ punctures, the following holds true: There exists a complete hyperbolic metric $\hat{g}_{\infty}$ on $\hat{\Sigma}:=\Sigma^{(1)}$ and diffeomorphisms $\hat{f}_{j}: \hat{\Sigma} \rightarrow \Sigma^{(j)}$ so that

$$
\hat{f}_{j}^{*} g^{(j)} \rightarrow \hat{g}_{\infty} \text { smoothly locally on } \hat{\Sigma}=\Sigma^{(1)}
$$

and so that the diffeomorphisms $\hat{f}_{j}$ map $\gamma_{i}^{(1)}$ to $\gamma_{i}^{(j)}, i \geq \kappa_{1}+1$, while a neighbourhood of each puncture (respectively, of each pair of punctures) of $\left(\Sigma^{(1)}, g_{\infty}\right)$ obtained by collapsing one the $\gamma_{i}^{(1)}, 1 \leq i \leq \kappa_{1}$ (respectively, one of the $\sigma_{i}^{(1)}$ ) is mapped to a neighbourhood of the corresponding puncture (respectively, pair of punctures) of $\Sigma^{(j)}$.

In addition, for $j$ sufficiently large, we can modify these diffeomorphisms as described in the proof of Claim 3 on p. 75 of [11] to ensure that $\hat{f}_{j}:\left(\hat{\Sigma}, g_{\infty}\right) \rightarrow$ $\left(\Sigma^{(j)}, g^{(j)}\right)$ is given in a neighbourhood of $\bigcup_{i=\kappa_{1}+1}^{k} \gamma_{i}^{(1)}$ by the identity in the respective Fermi coordinates.

In slight abuse of notation we now denote by $\mathrm{C}^{+}(\ell), \ell \geq 0$, the unique hyperbolic half-collar which has one boundary curve of constant geodesic curvature and length $L$, where $L^{2}-\ell^{2}=d^{2}$, while the other boundary curve is a geodesic of length $\ell$ if $\ell>0$, respectively, degenerated to a hyperbolic cusp if $\ell=0$. We then construct the limit surface $\left(\Sigma, g_{\infty}\right)$ out of the limiting surface $\left(\hat{\Sigma}, \hat{g}_{\infty}\right)$ with geodesic boundary obtained above and the half-collars $\mathrm{C}^{+}\left(\ell_{i}^{\infty}\right), i=1, \ldots, k$, by gluing the non-degenerate halfcollars $\mathrm{C}^{+}\left(\ell_{i}^{\infty}\right), i \geq \kappa_{1}+1$, to $\hat{\Sigma}$ along the corresponding non-collapsed boundary curves of $\left(\hat{\Sigma}, \hat{g}_{\infty}\right)$, and adding the degenerate collars $\mathrm{C}^{+}\left(\ell_{i}^{\infty}\right), i \leq \kappa_{1}$, as additional connected components of $\left(\Sigma, g_{\infty}\right)$. As the connected components of $M \backslash \bigcup_{i=1}^{\kappa_{1}} \gamma_{i}^{(j)}$ are given by $M \backslash \bigcup_{i=1}^{\kappa_{1}} \mathrm{C}^{+}\left(\ell_{i}^{(j)}\right)$ and a collection $\left\{\mathrm{C}^{+}\left(\ell_{i}^{(j)}\right)\right\}_{i=1}^{\kappa_{1}}$ of degenerating halfcollars, we can now extend the diffeomorphisms $\hat{f}_{j}$ obtained above to the required diffeomorphisms

$$
f_{j}: \Sigma \rightarrow M \backslash\left(\bigcup_{i=1}^{\kappa_{1}} \gamma_{i}^{(j)} \cup \bigcup_{i=1}^{\kappa_{2}} \sigma_{i}^{(j)}\right)
$$


as follows: The degenerated connected components $\mathrm{C}^{+}\left(\ell_{i}^{\infty}=0\right), 1 \leq i \leq \kappa_{1}$, which are isometric to $\left([0, \infty) \times S^{1}, \rho_{\ell=0}^{2}\left(d s^{2}+d \theta^{2}\right)\right), \rho_{0}(s)=\frac{1}{d+s}$, are mapped to the degenerating half-collars $\mathrm{C}^{+}\left(\ell_{i}^{(j)}\right)$ in $\left(M, g^{(j)}\right)$ which are bounded by $\gamma_{i}^{(j)}$ and $\Gamma_{i}^{(j)}$ with $f_{j}$ chosen so that it is given in collar coordinates by a bijection from $[0, \infty) \times S^{1}$ to $\left(0, \bar{X}_{d}\left(\ell_{i}^{(j)}\right)\right] \times S^{1}$ with $f_{j}(s, \theta)=\left(\bar{X}_{d}\left(\ell_{i}^{(j)}\right)-s, \theta\right)$ on domains which exhaust $[0, \infty) \times S^{1}$, say for $s \in\left[0, \frac{1}{2} \bar{X}_{d}\left(\ell_{i}^{(j)}\right)\right]$. As $\rho_{\ell}\left(\bar{X}_{d}\left(\ell_{i}^{(j)}\right)-\cdot\right) \rightarrow \rho_{0}(\cdot)$ locally uniformly on $[0, \infty)$ as $\ell \rightarrow 0$, this ensures that the pulled back metrics converge on every compact subset of these connected components of the limit surface as required.

Finally we extend $f_{j}$ to the collars that we glued to $\hat{\Sigma}$ as follows: We let $w_{i}^{+, \infty}$ and $w_{i}^{+,(j)}, i \geq \kappa_{1}+1$, be the width of the half-collars $\mathrm{C}^{+}\left(\ell_{i}^{\infty}\right)$ and $\mathrm{C}^{+}\left(\ell_{i}^{(j)}\right)$, i.e. the geodesic distance between the two boundary curves, and note that $w_{i}^{+,(j)} \rightarrow w_{i}^{+, \infty}$ since $\ell_{i}^{(j)} \rightarrow \ell_{i}^{\infty}$. We may thus choose smooth bijections $\phi_{i}^{(j)}:\left[0, w_{i}^{\infty}\right] \rightarrow\left[0, w_{i}^{(j)}\right]$ which agree with the identity in a neighbourhood of 0 and converge to the identity as $j \rightarrow \infty$. Since $\hat{f}_{j}: \hat{\Sigma} \rightarrow \hat{\Sigma}^{(j)}$ is given by the identity in Fermi coordinates near the boundary curves, we may extend $\hat{f}_{j}$ to a smooth diffeomorphism $f_{j}$ on $\Sigma$ for which $f_{j}^{*} g^{(j)}$ converges as claimed in Theorem 1.2 by defining $f_{j}$ on $\mathrm{e}^{+}\left(\ell_{i}^{\infty}\right)$ by $f_{j}(x, \theta)=\left(\phi_{i}^{(j)}(x), \theta\right)$ in Fermi coordinates.

Open Access This article is licensed under a Creative Commons Attribution 4.0 International License, which permits use, sharing, adaptation, distribution and reproduction in any medium or format, as long as you give appropriate credit to the original author(s) and the source, provide a link to the Creative Commons licence, and indicate if changes were made. The images or other third party material in this article are included in the article's Creative Commons licence, unless indicated otherwise in a credit line to the material. If material is not included in the article's Creative Commons licence and your intended use is not permitted by statutory regulation or exceeds the permitted use, you will need to obtain permission directly from the copyright holder. To view a copy of this licence, visit http://creativecommons.org/licenses/by/4.0/.

\section{Appendix}

We finally include a proof of Remark 2.4, which uses that the Dirichlet energy $E(u)=\frac{1}{2} \int|d u|_{g}^{2} d v_{g}$ is conformally invariant and hence well defined for functions on Riemann surfaces.

Proof of Remark 2.4 If $M$ has at least two boundary curves, we use that the harmonic function $\bar{f}$ which is 1 on $\Gamma_{i}$ and zero on all other boundary components, minimises $E$ among all functions with the same Dirichlet boundary data. Given a cylindrical neighbourhood of $\Gamma_{i}$ as in the remark, we thus have $E(f)=\frac{\pi}{Z} \geq \delta:=E(\bar{f})$ for the function $f$ which is linear on $U \sim(0, Z] \times S^{1}$ and zero elsewhere, and thus $Z \leq \frac{\pi}{\delta}$.

If $M$ has only one boundary curve, we fix instead some curve $\sigma$ with distinct endpoints on the boundary curve $\Gamma$ so that $\sigma$ is homotopically non-trivial with respect to variations by curves with endpoints on $\Gamma$. Then there exists a simple closed curve $\gamma$ in the interior of $M$ so that any curve $\sigma^{\prime}$ which is homotopic to $\sigma$ (with endpoints on $\Gamma)$ must intersect any curve $\gamma^{\prime}$ that is homotopic to $\gamma$. We claim that there exists some 
$\delta>0$ so that $E(f) \geq \delta$ for all functions $f: M \rightarrow \mathbb{R}$ which are equal to 1 on $\Gamma$ and for which there is a curve $\gamma^{\prime}$ homotopic to $\gamma$ so that $\left.f\right|_{\gamma^{\prime}} \leq 0$. As in the first case, this will then imply that $Z \leq \frac{\pi}{\delta}$. To prove the claim, we fix a neighbourhood $V$ of the fixed curve $\sigma$ that is diffeomorphic (but not necessarily conformal) to some rectangle $R=[-c, c] \times[-b, b]$, say with $\sigma$ corresponding to $\{0\} \times[-b, b]$ and with $V \cap \Gamma$ corresponding to $[-c, c] \times\{-b\} \cup[-c, c] \times\{b\}$ for the chosen diffeomorphism $\phi: R \rightarrow V$ and fix some smooth metric $g$ on $M$ that is compatible to $c$. Using that $\phi^{*} g$ is equivalent to the Euclidean metric on $R$ we obtain that there exists $c_{0}>0$ (allowed to depend on the above construction) so that for any $f: M \rightarrow \mathbb{R}$ as considered above and $\tilde{f}:=f \circ \phi$

$$
\begin{aligned}
E(f) & \geq \frac{1}{2} \int_{R}|d \tilde{f}|_{\phi^{*} g}^{2} d v_{\phi^{*} g} \geq c_{0} \int_{R}\left|\partial_{x} \tilde{f}\right|^{2}+\left|\partial_{y} \tilde{f}\right|^{2} d x d y \\
& \geq c_{0} c b^{-1} \inf _{a \in[-c, c]}\left(\int_{\{a\} \times[-b, b]}\left|\partial_{y} \tilde{f}\right| d y\right)^{2} .
\end{aligned}
$$

As the curves $\phi(\{a\} \times[-b, b]), a \in[-c, c]$ are homotopic to $\sigma$ and thus intersect the curve $\gamma^{\prime}$ for which $\left.f\right|_{\gamma^{\prime}} \leq 0$, while $\tilde{f}(a, \pm b)=1$, we thus get $E(f) \geq \delta:=$ $4 c_{0} c b^{-1}>0$ as claimed.

\section{References}

1. Berger, M.S.: Riemannian structures of prescribed Gaussian curvature for compact 2-manifolds. J. Differ. Geom. 5, 325-332 (1971)

2. Borer, F., Galimberti, L., Struwe, M.: "Large" conformal metrics of prescribed Gauss curvature on surfaces of higher genus. Comment. Math. Helv. 90, 407-428 (2015)

3. Brendle, S.: Curvature flows on surfaces with boundary. Math. Ann. 324, 491-519 (2002)

4. Brendle, S.: A family of curvature flows on surfaces with boundary. Math. Z. 241, 829-869 (2002)

5. Buzano, R., Rupflin, M.: Smooth long-time existence of harmonic Ricci flow on surfaces. J. Lond. Math. Soc. 95, 277-304 (2017)

6. Chang, S.Y.A., Yang, P.: Conformal deformation of metrics on $S^{2}$. J. Differ. Geom. 27, 259-296 (1988)

7. Chen, W., Li, C.: Gaussian curvature in the negative case. Proc. Am. Math. Soc. 131, 741-744 (2003)

8. Cherrier, P.: Problèmes de Neumann non linéaires sur les variéetés riemanniennes. J. Funct. Anal. 57, 154-206 (1984)

9. Ding, W., Li, J., Liu, Q.: Evolution of minimal torus in Riemannian manifolds. Invent. Math. 165, 225-242 (2006)

10. Eells, J., Sampson, J.H.: Harmonic mappings of Riemannian manifolds. Am. J. Math. 86, 109-160 (1964)

11. Hummel, C.: Gromov's Compactness Theorem for Pseudo-holomorphic Curves. Progress in Mathematics, vol. 151. Birkhäuser Verlag, Basel (1997)

12. Krantz, S., Parks, H.: The Implicit Function Theorem, History, Theory and Applications. Birkhäuser Boston Inc., Boston, MA (2002)

13. Kazdan, J., Warner, F.W.: Curvature functions for compact 2-manifolds. Ann. Math. 99, 14-47 (1974)

14. Li, X., Liu, P.: A Moser-Trudinger inequality on the boundary of a compact Riemann surface. Math. Z. 250, 363-386 (2005)

15. Mazzeo, R., Taylor, M.: Michael curvature and uniformization. Israel J. Math. 130, 323-346 (2002)

16. Müller, R.: Ricci flow coupled with harmonic map flow. Ann. Sci. Éc. Norm. Supér. 45, 101-142 (2012)

17. Osgood, B., Phillips, R., Sarnak, P.: Extremals of determinants of Laplacians. J. Funct. Anal. 80, 148-211 (1988)

18. Randol, B.: Cylinders in Riemann surfaces. Comment. Math. Helv. 54, 1-5 (1979)

19. Rupflin, M., Topping, P.M.: Flowing maps to minimal surfaces. Am. J. Math. 138, 1095-1115 (2016) 
20. Rupflin, M., Topping, P.M.: Teichmüller harmonic map flow into nonpositively curved targets. J. Differ. Geom. 108, 135-184 (2018)

21. Rupflin, M., Topping, P.M.: Global weak solutions of the Teichmüller harmonic map flow into general targets. Anal. PDE 12, 815-842 (2019)

22. Rupflin, M., Topping, P.M.: Horizontal curves of hyperbolic metrics. Calc. Var. Partial Differ. Equ. 57, Art. 106 (2018)

23. Rupflin, M.: Teichmüller harmonic map flow from cylinders. Math. Ann. 368(3-4), 1227-1276 (2017)

24. Schoen, R., Yau, S.T.: Lectures on Differential Geometry. International Press, Cambridge, MA (1994)

25. Struwe, M.: A flow approach to Nirenberg's problem. Duke Math. J. 128(1), 19-64 (2005)

26. Trudinger, N.: On imbeddings into Orlicz spaces and some applications. J. Math. Mech. 17, 473-483 (1967)

Publisher's Note Springer Nature remains neutral with regard to jurisdictional claims in published maps and institutional affiliations. 